\title{
Diversidade de espécies dos gêneros de Crematogaster, Gnamptogenys e Pachycondyla (Hymenoptera: Formicidae) e complementaridade dos métodos de coleta durante a estação seca numa estação ecológica no estado do Pará, Brasil.
}

\author{
Jorge Luiz Pereira SOUZA ${ }^{1}$, C.A.R. MOURA ${ }^{1}$, Ana Y. HARADA ${ }^{2}$, Elizabeth FRANKLIN ${ }^{1}$
}

\section{RESUMO}

As formigas são muito usadas em programas de inventários e monitoramentos, resultando em grande quantidade de material coletado com vários métodos, limitaçôes financeiras e na falta de taxonomos para vários táxons. Para contribuir na elaboração de protocolos alternativos, analisamos a complementaridade de dois métodos de amostragem na detecção da riqueza de espécies. Em seis parcelas de $1 \mathrm{~km}^{2}$ distribuídas em 33.000 ha de floresta primária, investigamos a diversidade e a freqüência das espécies de Crematogaster, Gnamptogenys e Pachycondyla, como parte do protocolo de formigas de serapilheira do Projeto TEAM. Em cada parcela, quatro transectos de $100 \mathrm{~m}$ foram instalados. Dez sub-amostras foram efetuadas por transecto, usando extrator de Winkler e armadilha de fosso. Registramos 36 espécies, sendo 15 novos registros para o Pará e 29 para a área de estudo. Dezessete espécies foram comuns aos dois métodos, onze foram capturadas com armadilhas de fosso e oito com Winkler. As proporçōes de espécies representadas por um indivíduo ("singletons") foram altas para ambos os métodos (>0,4). Dezenove espécies foram coletadas por apenas um método e três foram representadas por apenas um indivíduo em cada método. A análise de ordenação (escalonamento multidimensional semi-híbrido) revelou diferenças na estrutura das comunidades de formigas obtidas pelos dois métodos de amostragem. Houve um efeito de complementaridade entre os dois métodos para a obtenção de um levantamento mais completo da riqueza de espécies, significando que ambos devem ser usados no protocolo. Outros recursos, como a rarefação e/ou diluição das amostras são recursos a serem avaliados em futuras investigaçỗes.

PALAVRAS-CHAVE: Formigas, extrator de Winkler, armadilha de fosso, Pittfall, Amazonia.

\section{Diversity of species of the genera Crematogaster, Gnamptogenys and Pachycondyla, (Hymenoptera: Formicidae) and complementarity of sampling methods during the dry season in an ecological station in the Brazilian state of Pará.}

\section{ABSTRACT}

Ants are largely used for monitoring and inventory programs, resulting in great amounts of material sampled with several methodologies, financial limitations and lack of experts to do the taxonomy of many taxa. To contribute for the elaboration of alternative protocols, we analyzed the complementarity of two sampling methods in the detection of species richness. In 6 plots of $1 \mathrm{~km}^{2}$ distributed in an area of 33,000 ha of continuous primary forest, in the Brazilian state of Pará, the diversity and the frequency of species of genera Crematogaster, Gnamptogenys and Pachycondyla were investigated, as part of the "Ant methodology Protocol" of the Project TEAM. Using the Winkler extractor and pitfall traps, 10 sub-samples were collected per transect. In each plot, 4 transects of $100 \mathrm{~m}$ were installed. We registered 36 species, and 15 are new records for the state, and 29 for the study site. Seventeen species were common to both sampling methods, 11 were captured with pitfall and 8 with Winkler. The proportions of singletons were high for both methods $(>0,4)$. Nineteen species were captured by only one method and three were represented by only one individual in both methods. Ordination analysis (SSH, Semi-Strong Hybrid Multi-Dimensional Scaling) indicated differences in community structure between both sampling methods. There was a complementarity's effect between these two methods in order to obtain a more complete inventory of the species richness, indicating that both must be used in the protocol. Other resources like rarefaction and/or sample dilution must be evaluated in future investigations.

KEYWORDS: Ants, Winkler extractor, Pitfall traps, Amazon.

${ }^{1}$ Instituto Nacional de Pesquisas da Amazônia INPA, CPEN, CP 478, 69011-970.

${ }_{2}^{2}$ Museu Paraense Emílio Goeldi, MPEG, CP 399, Belém, PA., Brasil. 


\section{INTRODUÇÃO}

Nos trópicos, os invertebrados constituem aproximadamente 94\% da biomassa animal, sendo as formigas uma presença evidente em grande parte dos ecossistemas terrestres (Longino et al., 2002; Ellwood e Foster, 2004). Esses animais exercem grande influência no solo (Lobry de Bruyn, 1999), possuem ampla distribuição, alta riqueza de espécies e são relativamente fáceis de identificar (Hölldobler e Wilson, 1990; Agosti et al., 2000). As muitas espécies que nidificam em serapilheira constroem ninhos efêmeros, que são considerados sensíveis a modificaçôes no habitat (Byrne, 1994).

Dada a importância deste grupo, seu uso em monitoramentos e inventários rápidos de biodiversidade ("BioRap Methodology" de acordo com Margules e Redhead, 1995) foi ampliado pelo mundo e na Amazônia, principalmente em estudos de grande escala espaço-temporal, como os projetos "Dinâmica Biológica de Fragmentos Florestais - PDBFF" e "Tropical Ecology Assesment and Monitoring - TEAM" (Carvalho e Vasconcelos, 1999; Batra, 2003) e PPBio. Esse estudo faz parte do Protocolo de Formigas de Serapilheira (AMP) do Projeto TEAM Caxiuanã, que utiliza o extrator de Winkler e a armadilha de fosso ("pitfall traps"), dois métodos amplamente utilizados para a coleta de formigas.

Por outro lado, a ampliação do uso destes organismos implica em uma crescente quantidade de material coletado por mais de um método de coleta, o que vai de encontro à limitação de recursos de ordem financeira e número insuficiente de pessoal treinado na taxonomia de muitos grupos. Segundo Danielsen et al. (2003), em países em desenvolvimento os custos podem vir a ser tão altos que torna-se improvável que o projeto continue por mais do que alguns anos. Para programas de monitoramento de longo prazo, o desenho do protocolo deve ser considerado cuidadosamente, deve focar em poucos grupos ou guildas e em poucos métodos de amostragem absolutamente práticos (Sörensen et al., 2002). As técnicas aplicadas devem ser realísticas, rápidas, passíveis de serem repetidas e viáveis quanto ao seu custo-benefício (Margules e Austin, 1991; Kim, 1993; Fisher, 1999; Parr e Chow, 2001). O estabelecimento dessas amostragens padronizadas é especialmente importante nos trópicos, onde a riqueza de espécies é alta e o conhecimento científico sobre a fauna ainda é modesto (Umetsu et al., 2006). Portanto, uma ênfase maior deve ser dada para as análises de redução do esforço de coleta nos protocolos.

Um ponto de partida para a redução do esforço de coleta, triagem e identificação, seria analisar a possibilidade do uso de apenas um método de coleta. As armadilhas de fosso foram mais eficientes, produtivas e consistentes que o extrator de Winkler em sistema de savana para a coleta de formigas epigêicas (Parr e Chown, 2001). Por outro lado, o extrator de Winkler tem sido recomendado para áreas de floresta com maior camada de serapilheira e tem sido muito usado por diversos autores no Brasil (Majer et al., 1997; Delabie et al., 2000a; Vasconcelos e Delabie, 2000; Silva e Silvestre, 2004).

Em outras regiōes, o efeito complementar na coleta de formigas da serapilheira já foi registrado por Olson (1991) ao comparar a eficácia do extrator de Winkler e da armadilha de fosso. Isto significa que as informaçóes obtidas com o uso destas armadilhas foram complementares para a obtenção de um levantamento mais completo da riqueza de gêneros e espécies. Os objetivos deste estudo foram 1) investigar a diversidade e a freqüência das espécies pertencentes aos gêneros Crematogaster, Gnamptogenys e Pachycondyla e 2) verificar se dois métodos de amostragem (armadilhas de fosso e extrator de Winkler) se complementam na detecção da diversidade da área estudada na estação científica em Caxiuanã, visando contribuir para a elaboração de protocolos mais simples e eficientes.

\section{MATERIAL E MÉTODOS}

As coletas foram efetuadas nas parcelas permanentes do Projeto TEAM - Caxiuanã, como parte do Protocolo de Formigas de Serapilheira ("Ants Methodology Protocol AMP”). Estas parcelas estão alocadas na Estação Científica Ferreira Penna (ECFPn), do Museu Paraense Emílio Goeldi (MPEG). A estação tem uma extensão aproximada de 33.000 ha e forma parte da Floresta Nacional de Caxiuanã, localizada

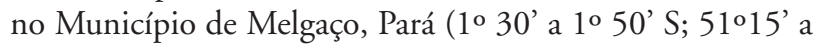
51045' L) (Lacruz, 1996). A escala espacial empregada foi de seis parcelas de $1 \mathrm{~km}^{2}$ cada, totalizando 600 ha, dotadas de um sistema de trilhas que divide a parcela em 100 sub-parcelas de 1 ha cada. Os solos predominantes são Latossolos Amarelos, em geral bem drenados, com estrutura maciça e baixa fertilidade natural (Kern \& Costa, 1994 apud Lacruz, 1996). O clima local é tropical úmido, "Am" na classificação de Köppen, sendo registrada uma precipitação média de $60 \mathrm{~mm} / \mathrm{mês}$ nos meses de Outubro e Novembro, os meses mais secos. A tipologia dominante é classificada como Floresta Ombrófila densa de terra firme (Almeida \& Thales, 2003).

Dois métodos de amostragem foram utilizados: extrator de Winkler e armadilhas de fosso (pitfall traps) (Bestelmeyer $e t$ al., 2000). A coleta foi realizada na estação seca, entre os dias 26/10/2003 e 3/11/2003 no horário de 9:00 às 15:00. Vinte e quatro transectos foram delimitados e coletados. A unidade amostral foi o transecto, composto por 10 sub-amostras espaçadas 10 metros entre si totalizando 240 amostras por método de coleta.

O extrator de Winkler método é apropriado para usar em ambientes de floresta, para coletar formigas que utilizem a serapilheira para forragear ou para abrigar-se (Bestelmeyer 
et al. 2000), e foi considerado o mais eficiente em numero de espécies coletadas (Delabie et al. 2000b., Fisher et al. 2000). Neste estudo a área de serapilheira peneirada foi de $1 \mathrm{~m}^{2}$, e esta permaneceu nos extratores por 48 horas, tempo suficiente para amostras qualitativas de espécies de formigas (Majer $e t$ al. 1997). O volume de serapilheira variou de 3,0 a 15,0 L. e sua média foi de $8,9 \mathrm{~L}$.

A armadilha de fosso, instalada ao nível do solo, é útil para coletar formigas predadoras e necrófagas como as poneromorfas, membros da subfamília Ponerinae. Em outras regiōes, esta armadilha age de maneira complementar ao extrator de Winkler, uma vez que coleta as formigas maiores que não passam pela peneira e pelo recipiente de extração do extrator (Olson, 1991). Os possíveis erros de coleta e interpretação dos dados obtidos da armadilha de fosso são discutidos por Adis (1979). Os dados gerados pela armadilha de fosso incluem riqueza e composição das espécies ativas na serapilheira durante o período de coleta (Bestelmeyer $e t$ al. 2000). Entretanto, a maioria dos estudos (Olson, 1991; Majer et al., 1997; Vasconcelos et al., 2003) utiliza períodos em torno de 48 horas, tempo também adotado por nosso estudo. As armadilhas de fosso foram instaladas aproximadamente a $20 \mathrm{~cm}$ de distância de onde foi retirada a serapilheira para a coleta do extrator de Winkler. Suas dimensōes de cerca de 10 $\mathrm{cm}$ de diâmetro e $10 \mathrm{~cm}$ de profundidade (Lutinski e Garcia, 2005), foram preenchidas em aproximadamente $1 / 3$ de seu volume com álcool etílico (van den Berghe, 1992) a 70\% ao qual adicionamos cerca de 10 gotas de detergente neutro por litro de solução para quebrar a tensão superficial do álcool e facilitar a morte e preservação da fauna coletada.

Os indivíduos dos gêneros Crematogaster, Gnamptogenys e Pachycondyla foram morfotipados e, quando possível, identificados em nível taxonômico de espécie com auxílio das chaves de Longino (2003), Lattke $(1990,1995)$ e Brown modificada (MacKay e MacKay 2003). Uma coleção de referência está depositada na coleção entomológica do INPA e o restante do material está depositado na coleção do MPEG.

\section{TRATAMENTO ESTATÍSTICO DOS DADOS}

Foi efetuado o teste o $\mathrm{t}$ pareado utilizando o número total de espécies, para verificar se haveria diferença entre o número de espécies capturadas em cada método, utilizando o pacote estatístico SYSTAT 10 (Wilkinson, 1990). Para verificar se a composição da comunidade de espécies de formigas seria diferente entre os dois métodos, a técnica de ordenação foi usada para reduzir a variação da comunidade de formigas em duas dimensões, através do escalonamento multidimensional semi-híbrido (SSH, Semi-Strong Hybrid Multi-Dimensional Scaling) no programa PATN (Belbin, 1992). A decisão para reduzir a comunidade a dois eixos foi feita a priori e as ordenaçôes foi feita para os dados qualitativos (presença/ausência), devido ao uso do forrageio em massa e a distribuição dos ninhos na serapilheira (Levings e Franks 1982; Hölldobler e Wilson 1990; Soares et al. 2001, Longino et al. 2002). A ordenação foi baseada em dados qualitativos para dar mais peso para as espécies incomuns, uma vez que as comuns tendem a ocorrer em todos os locais. O índice de Sörensen foi usado para indicar a dissimilaridade entre as amostras. Utilizando o SYSTAT 10, foi feita análise de variância para testar o efeito do método de coleta sobre a composição da comunidade coletada.

\section{RESULTADOS}

\section{RIQUEZA DE ESPÉCIES}

No total dos dois métodos de coleta combinados, foram coletadas 36 espécies de formigas pertencentes aos gêneros Crematogaster, Gnamptogenys e Pachycondyla. Crematogaster foi representado por sete espécies, sendo uma espécie nova, provisoriamente designada por "C. JTL 017", Gnamptogenys por dez espécies definitivamente identificadas e por quatro morfoespécies e Pachycondyla por treze espécies e duas morfoespécies (Tabela 1). Considerando o total das subamostras (480), C. tenuicula e C. levior foram as espécies mais freqüentes, sendo registradas em $26 \%$ e $23 \%$ das subamostras, respectivamente.

As proporçôes de espécies freqüentes e não freqüentes, se aproximaram nos resultados dos dois métodos de coleta, sendo que as proporçôes de "singletons" foram muito altas para ambos os métodos $(>0,4)$ (Tabela 2). Onze espécies foram capturadas exclusivamente com a armadilha de fosso e 8 com extrator de Winkler, representando proporçôes muito altas em ambos os métodos $(>0,25)$. Poucas espécies (3) foram representadas por apenas um indivíduo em cada método de amostragem ("singletons verdadeiras"). Dezessete espécies foram comuns aos dois métodos (Tabela 2).

Comparativamente, as riquezas de espécies coletadas nos 24 transectos pelos dois métodos de amostragem se aproximam (média \pm desvio padrão ; armadilhas de fosso: $5,95 \pm 1,82$; Winkler: $5,42 \pm 1,74 ; \mathrm{t}=1,248 ; \mathrm{df}=23 ; \mathrm{P}=0,200)$. A análise de ordenação detectou diferenças na composição de espécies das comunidades de formigas entre os dois métodos (MANOVA; Pillai Trace $=3,942 ; \mathrm{P}=0,034)$. Esta diferença é uma premissa para haver uma complementaridade entre os métodos. A variação foi encontrada no eixo SSH2 (ANOVA; SSH2: F 1,23 = 7,114; P = 0,014). Não houve diferença no eixo SSH1 (ANOVA; SSH1: F 1,23 =0,877; P = 0,359). Apesar da sobreposição de alguns pontos, as comunidades coletadas pelo extrator de Winkler estão mais próximas entre si e possuem distribuição mais agregada em comparação com a fauna coletada pela armadilhas de fosso, que mostrou uma distribuição mais dispersa (Figura 1). 
Tabela 1 - Abundância relativa das espécies de Crematogaster, Gnamptogenys e Pachycondyla coletadas na serapilheira em 24 parcelas, com armadilhas de fosso e extrator de Winkler. Valores obtidos representam o número de presenças observado dividido pelo número de subamostras ( $n=240$ por método de coleta). As espécies estão ordenadas de acordo com o gênero e em ordem decrescente de frequência.

\begin{tabular}{lcccc}
\hline \multirow{2}{*}{ Espécies } & \multicolumn{2}{c}{$\begin{array}{c}\text { Armadilha de } \\
\text { Fosso }\end{array}$} & \multicolumn{2}{c}{$\begin{array}{c}\text { Extrator de } \\
\text { Winkler }\end{array}$} \\
\cline { 2 - 5 } & $\%$ & Presença & $\%$ & Presença \\
\hline
\end{tabular}

\section{Crematogaster}

\begin{tabular}{lcccc} 
C. tenuicula (Forel, 1904) & 25 & 60 & 27,9 & 67 \\
\hline C. levior (Longino, 2003) & 18,3 & 44 & 28,8 & 69 \\
\hline C. sotobosque (Longino, 2003) & 4,2 & 10 & 7,5 & 18 \\
\hline C. brasiliensis (Mayr, 1878) & 2,5 & 6 & 4,2 & 10 \\
\hline C. flavosensitiva (Longino, 2003) & 0,8 & 2 & 3,3 & 8 \\
\hline C. nigripilosa (Mayr, 1870) & 0,0 & 0 & 0,8 & 2 \\
\hline C. "JTL 017 ( & 0,4 & 1 & 0,0 & 0 \\
\hline
\end{tabular}

\section{Gnamptogenys}

\begin{tabular}{lcccc}
\hline G. tortuolosa (F. Smith, 1858) & 17,9 & 22 & 1,3 & 3 \\
\hline G. horni (Santschi, 1929) & 9,2 & 7 & 6,3 & 15 \\
\hline G. sp.4 & 2,9 & 5 & 0,4 & 1 \\
\hline G. moelleri (Forel, 1912) & 2,1 & 1 & 1,7 & 4 \\
\hline G. relicta (Mann, 1916) & 0,4 & 1 & 3,3 & 8 \\
\hline G. triangularis (Mayr, 1887) & 0,4 & 1 & 0,4 & 1 \\
\hline G. sp.2 & 0,4 & 0 & 0,4 & 1 \\
\hline G. hartmanni (Wheeler, 1915) & 0,0 & 0 & 0,4 & 1 \\
\hline G. mediatrix (Brown, 1958) & 0,0 & 0 & 0,4 & 1 \\
\hline G. striatula (Mayr, 1883) & 0,0 & 1 & 0,8 & 2 \\
\hline G. annulata (Mayr, 1887) & 0,4 & 1 & 0,0 & 0 \\
\hline G. haenschi (Emery, 1902) & 0,4 & 1 & 0,0 & 0 \\
\hline G. sp.1 & 0,4 & 1 & 0,0 & 0 \\
\hline G. sp.3 & 0,4 & 1 & 0,0 & 0 \\
\hline Pachycondyla & & & & \\
\hline P. constricta (Mayr, 1883) & 5,8 & 14 & 7,9 & 19 \\
\hline P. harpax (Fabricius, 1804) & 6,3 & 15 & 3,3 & 8 \\
\hline P. obscuricornis (Emery, 1890) & 6,7 & 16 & 0,0 & 0 \\
\hline P. apicalis (Latreille, 1802) & 5,4 & 13 & 0,0 & 0 \\
\hline P. arhuaca (Forel,1901) & 3,3 & 8 & 2,1 & 5 \\
\hline P. crassinoda (Latreille, 1802) & 1,3 & 3 & 0,0 & 0 \\
\hline P. unidentata (Mayr, 1862) & 0,0 & 0 & 1,3 & 3 \\
\hline P. ferruginea (F. Smith) & 0,4 & 1 & 0,4 & 1 \\
\hline P. impressa (Emery, 1901) & 0,4 & 1 & 0,4 & 1 \\
\hline & & & & \\
\hline
\end{tabular}

\begin{tabular}{lllll}
\hline P. magnifica (Borgmeier, 1929) & 0,4 & 1 & 0,0 & 0 \\
\hline P. commutata (Roger, 1860) & 0,4 & 1 & 0,0 & 0 \\
P. sp. 2 & 0,4 & 1 & 0,0 & 0 \\
\hline P. crenata (Roger, 1861) & 0,0 & 0 & 0,4 & 1 \\
P. stigma (Fabricius, 1804) & 0,0 & 0 & 0,4 & 1 \\
P. sp. 1 & 0,0 & 0 & 0,4 & 1 \\
\hline
\end{tabular}

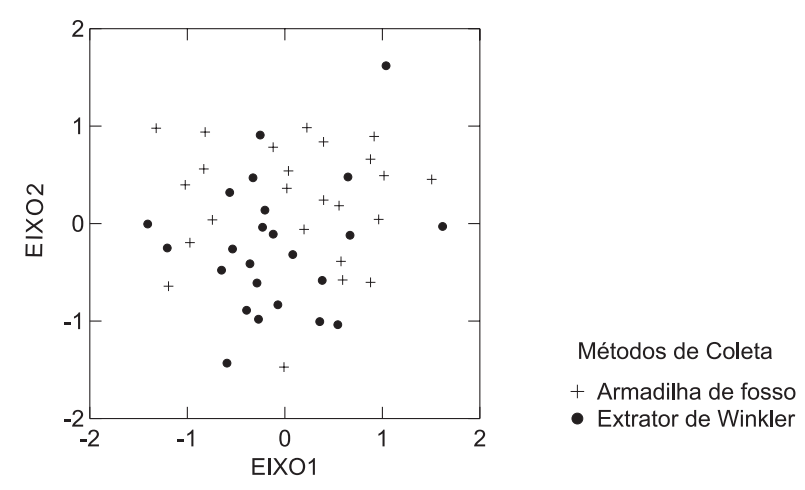

Figura 1. Ordenação em duas dimensões através do escalonamento multidimensional semi-híbrido (SSH) da comunidade de espécies de Crematogaster, Gnamptogenys e Pachycondyla, coletadas na serapilheira por dois métodos (armadilhas de fosso e extrator de Winkler) em 24 transectos de floresta primária.

\section{DISCUSSÃO}

Existem cerca de 102 espécies de Gnamptogenys em todo o globo, sendo 64 no Novo Mundo (Hedlund, 2004). A variação morfológica e comportamental destas formigas é pronunciada. Esse grupo geralmente nidifica na serapilheira e sobre troncos em decomposição e comumente são encontrados restos de artrópodes, incluindo outras formigas, nos ninhos de diversas espécies. A maioria das espécies é restrita às florestas tropicais úmidas (Lattke, 1990). Neste estudo, foram efetuados os primeiros registros de ocorrência no estado do Pará para as espécies G. haenschi, G. moelleri, G. triangularis e G. relicta (Kempf, 1972; Brandão, 1991). Além destas, G. annulata, G. mediatrix, G.striatula, G. tortuolosa são registradas pela primeira vez para estação científica de Caxiuanã. Alguns exemplares já foram coletados na Amazônia brasileira e encontram-se depositados na coleção de referência do Dr. Heraldo Vasconcelos, no INPA. G. hartmani possui registro no Brasil, estado da Bahia (Lattke, 1995). Na Venezuela foram registradas cerca de 27 espécies de Gnamptogenys (Brandão, 1991; Latke, 1995). A diversidade registrada na ECFPn corresponde a aproximadamente $50 \%$ desse total, significando que ao longo das coletas do protocolo de formigas do TEAM os registros da diversidade poderão aumentar na área. 
Tabela 2 - Riqueza de espécies e estimadores de frequência das espécies de Crematogaster, Gnamptogenys e Pachycondyla coletadas em armadilha de fosso e extrator de Winkler.

\begin{tabular}{|c|c|c|c|c|c|}
\hline & \multicolumn{2}{|c|}{ Armadilha de fosso } & \multicolumn{2}{|c|}{ Winkler } & \multirow{2}{*}{ Ambos } \\
\hline & Total & Proporção $0^{\mathrm{a}}$ & Total & Proporção ${ }^{\mathrm{a}}$ & \\
\hline Número de espécies observado & 28 & - & 25 & - & 36 \\
\hline Número de espécies frequentes (em mais que 10 subamostras) & 8 & 0,29 & 5 & 0,2 & - \\
\hline Número de espécies não frequentes (em menos que 10 subamostras) & 20 & 0,71 & 20 & 0,8 & - \\
\hline Espécies representadas por dois indivíduos ("Doubletons") & 1 & 0,04 & 2 & 0,08 & - \\
\hline Espécies representadas por um único indivíduo ("Singletons") & 14 & 0,5 & 10 & 0,4 & - \\
\hline $\begin{array}{l}\text { Espécies representadas por apenas um indivíduo nos dois métodos ("Singletons" } \\
\text { verdadeiras) }\end{array}$ & - & - & - & - & 3 \\
\hline Espécies encontradas em apenas um método (Específicas do método) & 11 & 0,39 & 8 & 0,29 & - \\
\hline Espécies comuns a ambos os métodos (Comuns) & - & - & - & - & 17 \\
\hline
\end{tabular}

a Relação entre o número de espécies e o número total de espécies.

O gênero Pachycondyla abriga uma grande diversidade morfológica e comportamental com cerca de 200 espécies descritas, com distribuição mundial (Wild, 2002). Os gêneros Mesoponera, Neoponera, Termitopone, Trachymesopus e Wadeura foram recentemente sinonimizados em Pachycondyla. No gênero Pachycondyla ocorrem desde espécies menores, criptobióticas que forrageiam na serapilheira (Wild, 2002) até as grandes e conspícuas, como $P$. apicalis, $P$. commutata, P. crassinoda, P. obscuricornis que foram coletadas apenas na armadilha de fosso. As espécies $P$. arhuaca, $P$. ferruginea e $P$. magnifica ainda não possuíam registro no estado do Pará (Kempf, 1972; Brandão, 1991). Somadas a estas, P. crenata, P. impressa e P. stigma são mencionadas pela primeira vez para Caxiuanã (A. Y. Harada, MPEG, comunicação pessoal). $P$. magnifica possui registros esparsos e com poucos indivíduos coletados, com exceção da localidade-tipo em Goiânia, Brasil. A ocorrência de uma operária também é relatada na localidade de Putumayo, Colômbia (Arias, 2003). Neste caso podemos estar diante de uma espécie rara ou sub-coletada, já que sua biologia é pouco conhecida.

O gênero Crematogasteré uma linhagem cosmopolita de Myrmicinae (Hölldobler e Wilson, 1990). A maioria das espécies é tropical, onde são elementos dominantes da fauna arbórea. Alguns grupos de espécies cruzaram a "linha de gelo", distribuindo-se pela zona temperada, onde nidificam com freqüência no solo e na superfície das pedras (Wheeler, 1906 apud Longino, 2003). As operárias são monomórficas e as castas possuem normalmente o mesmo tamanho. É conspícuo na maioria das faunas, especialmente nos trópicos, possuindo pequenas variaçôes morfológicas que variam local e regionalmente. Desse modo, foram gerados 889 nomes de espécies, incluindo sinonímias, havendo poucos trabalhos que sintetizem essa informação (Bolton, 1995; Longino, 2003). Dentre os nossos registros para Crematogaster, uma espécie nova foi detectada. Um maior esforço na divulgação de inventários e revisōes por meios eletrônicos e rede mundial de computadores poderia facilitar a confirmação destes e de novos registros (Scotland et al. 2003), já que algumas destas espécies já foram coletadas no Brasil e ainda estão esperando por divulgação.

Registramos uma alta incidência de espécies com um único registro ("singletons"), representando mais de $40 \%$ da fauna total amostrada para os três gêneros, nos dois métodos de amostragem. Algumas espécies que detectamos em baixa densidade na Estação Científica Ferreira Penna são comuns em outros estratos como: G. annulata, P. crenata, P. unidentata, que possuem hábitos arborícolas. G. haenschi, outra espécie com baixa densidade populacional, forrageia sobre troncos caídos (Latke, 1995; Longino, 2005). Populações pequenas são vulneráveis à extinção, mas com maior esforço de coleta elas podem perder o status de "singletons" (Margurran 2005). Murray \& Lepschi (2004), questionam se as espécies classificadas como "raras", não seriam abundantes em um local distinto do qual ela teria sido coletada a princípio. Este estudo dá uma resposta positiva a este questionamento, já que registramos espécies sabidamente abundantes em habitats distintos do qual investigamos nesse estudo. Portanto, a raridade destas espécies registradas em nosso estudo foi gerada pelos erros intrínsecos e aceitáveis dos métodos que empregamos na coleta, os quais se restringem à serapilheira e não prevêem coleta ativa. O termo "extraviado" ("stray", em inglês) utilizado por Longino (2005) é útil para definir essas espécies cujos hábitos as tornam raras em métodos de coleta não específicos, como as de hábitos arborícolas ou subterrâneos.

Numericamente, o número de espécies coletadas nos 24 transectos instalados em floresta primária não diferiu entre os dois métodos de amostragem, significando que estão coletando 
um número próximo de espécies ao longo de todos os 24 transectos. Contudo, em áreas de savana africana, a riqueza de espécies detectada foi maior usando armadilhas de fosso, embora o número de subamostras tenha sido menor que as efetuadas para o Winkler (Parr e Chown, 2001). Por outro lado, os dois métodos que utilizamos revelaram 36 espécies e foram complementares para uma avaliação da fauna da serapilheira. A diferença detectada pela análise de ordenação mostrou que os dois métodos coletam comunidades de formigas distintas, embora exista sobreposição possivelmente devido à atividade de forrageamento e de nidificação das espécies entre os dois métodos. Esse resultado reforçado pelo fato de que uma proporção relativamente alta de táxons específicos do método foi registrada tanto para o Winkler quanto para as armadilhas de fosso, a maioria com registros únicos ("singletons"), indicando que ambos os métodos contribuem para o inventário da comunidade de espécies na área. Essa sobreposição foi também detectada em savanas africanas por Parr e Chown (2001), que também detectaram maior número de espécies coletadas exclusivamente pelas armadilhas de fosso. A análise de ordenação também revelou que a fauna coletada pelo extrator de Winkler possui distribuição mais agregada em comparação com a fauna coletada pelas armadilhas de fosso.

O extrator de Winkler seleciona o tamanho da formiga de acordo com a malha utilizada e é mais eficiente na coleta de formigas pequenas e crípticas, com movimentação mais lenta devido ao hábito de forrageamento; a armadilha de fosso coleta as formigas usualmente maiores e mais ativas na área (Olson, 1991; Bestelmeyer et al., 2000; Parr \& Chown, 2001). Em termos de abundância relativa, o extrator de Winkler coleta maior número de formigas pequenas que as armadilhas de fosso (Olson, 1991; Parr e Chow, 2001). Essa complementaridade se aplica no caso de C. "JTL 017", $G$. annulata, G. haenschi, G. sp. 1, G. sp. 3, P. obscuricornis, P. apicalis, $P$. crassinoda, $P$. commutata e P.magnifica, que foram coletados apenas nas armadilhas de fosso. Essas espécies, exceto C. "JTL 017", são consideradas de grande porte (acima de $1 \mathrm{~cm}$ ). As espécies $C$. nigropilosa, $G$. hartmani, $G$. mediatrix, $G$. striatula, $P$. unidentata, $P$. crenata, $P$. stigma e $P$. sp. 1 só foram coletados no extrator de Winkler. Desse modo, além da importância da diversidade e da abundância, ficou claro que é necessário se considerar aspectos da biologia dos organismos estudados, em particular a mobilidade, como demonstrado por Brose \& Martinez (2004).

Uma vez que nenhum método registra todas as espécies de um grupo taxonômico presentes, inventários relativamente completos de comunidades de artrópodes com muitas espécies só são possíveis se métodos múltiplos e esforço extensivo forem empregados (Longino et al. 2002), Esse fato já foi também confirmado por Lindsey e Skinner (2001) em habitats semi- áridos no Sul da África, para a comunidade de formigas. Nossos resultados para os três gêneros estudados em Caxiuanã, Amazônia Oriental, confirmam o que já foi obtido por esses autores.

Os resultados mostraram que foi necessário usar tanto as armadilhas de fosso como os extratores de Winkler na coleta de formigas da serapilheira em florestas primárias na área deste estudo. Desse modo, uma vez que essas duas amostragens foram complementares, elas podem ser combinadas de modo a reduzir o esforço geral requerido para um completo inventário (Parr \& Chown, 2001). Portanto, as investigações visando a redução do esforço de coleta, triagem e análise dos dados devem continuar em futuros estudos, para uma melhor adequação dos protocolos de coleta, principalmente de grupos megadiversos como as formigas, Outros recursos, tais como rarefação e diluição de amostras podem também ser investigados para contribuir na elaboração de protocolos simples e eficientes. A rarefação visaria responder qual a quantidade mínima de amostras que deve ser coletada e triada e a diluição responderia qual a porcentagem máxima de diluição das mesmas para detectar as espécies de formigas que ocorrem no local do estudo. Deste modo, a combinação destes recursos poderia satisfazer a necessidade de informação sem reduzir a confiabilidade e a viabilidade dos dados necessários para revelar os padrões ecológicos nas taxocenoses (comunidades) em estudo.

\section{AGRADECIMENTOS}

Às Coordenaçôes da Estação Cientifica Ferreira Penna e do Projeto TEAM/Caxiuanã, pelo apoio financeiro e logístico. Ao Conselho Nacional de Desenvolvimento Científico e Tecnológico (CNPq) e à Fundação de Amparo a Pesquisa do Estado do Amazonas (FAPEAM) pelas bolsas de Mestrado. Às Coordenaçóes dos Cursos de Mestrado em Entomologia e em Ecologia, do PPGBTRN do convênio INPA/UFAM, Manaus, AM., pela oportunidade. Ao Dr. Thiago J. Izzo pelas inúmeras sugestôes nas análises estatísticas deste manuscrito.

\section{BIBLIOGRAFIA CITADA}

Adis, J. 1979. Problems of interpreting arthropod sampling with "Pitfall" traps. Zool. Anz., 202(3/4):177-184.

Agosti, D.; Majer, J.D.; Tennant, A.; Schultz, T.R. (Eds.). 2000. Ants: Standard methods for measuring and monitoring biodiversity. Smithsonian Institution Press, Washington, D. C., USA.

Almeida, S.S.; Thales, M.C. 2003. Tipos de vegetação da ECFPn, Caxiuanã, Município de Melgaço, Pará: uma primeira aproximação. In: Seminário Caxiuanã. Disponível em: http:// www.museu-goeldi.br/semicax/CBO_003.pdf. Acesso: $10 / 03 / 2005$. 
Arias, T.M. 2003. Nuevos registros de especies de hormigas de la subfamilia Ponerinae (Hymenoptera: Formicidae) para Colombia. Caldasia, 25(2):429-431.

Batra, P. 2003. Tropical Ecology, Assesment, and Monitoring (TEAM) Initiative: Ant Monitoring Protocol. 21pp.

Belbin, L. 1992. PATN: Pattern Analysis Package. CSIRO, Canberra, Australia.

Bestelmeyer, B.T.; Agosti, D.; Leeanne, F.; Alonso, T.; Brandão, C.R.F.; Brown, W.L.; Delabie, J.H.C.; Silvestre, R. 2000. Field techniques for the study of ground-living ants: An Overview, description, and evaluation. In: Agosti, D.; Majer, J.D.; Tennant, A.; Schultz, T.R. (Eds). Ants: Standard methods for measuring and monitoring biodiversity. Smithsonian Institution Press, Washington, DC., USA. p. 122-144.

Bolton, B. 1995. A New General Catalogue of the Ants of the World. Harvard University Press, Cambridge, MA, 504pp.

Brandão, C.R.F. 1991. Adendos ao Catálogo Abreviado das Formigas da Região Neotropical (Hymenoptera : Formicidae). Revista Brasileira de Entomologia, 35(2):319-412.

Brose, U.; Martinez, N.M. 2004. Estimating the richness of species with variable mobility. Oikos 105: 292-300.

Byrne, M.M. 1994. Ecology of twig-dwelling ants in a wet lowland tropical Forest. Biotropica, 26:61-72.

Carvalho, K.S.; Vasconcelos, H.L. 1999. Forest fragmentation in central Amazonia and its effects on litter-dwelling ants. Biological Conservation, 91:151-158.

Danielsen, F.; Mendoza, M.M.; Alviola, P.; Balete, D.S.; Enghoff, M.; Poulsen, M.K.; Jensen, A.C. (2003). Biodiversity monitoring in developing countries: what are we trying to achieve? Oryx, 37: 407-409.

Delabie, J.H.C.; Agosti, D.; Nascimento, I.C. 2000a. Litter ant communities of the Brazilian Atlantic rain forest region. In: Agosti, D.; Majer, J.D.; Alonso, L. e Schultz, T. (Eds.), Sampling ground-dwelling ants: case studies from de world's rain forests. Bulletin

18. Curtin University School of Environmental Biology, Perth, Australia, p.1-17.

Delabie, J.H.C.; Fisher, B.L.; Majer, J.D.; Wright, I.W. 2000 b. Sampling Effort and Choice of Methods. In: D. Agosti; Majer, J.D.; Tennant, A.; Schultz, T.R. (Eds). Ants: standard methods for measuring and monitoring biodiversity. Smithsonian Institution Press, Washington, DC., USA. p. 145-154.

Ellwood, M.D.F.; Foster, W.A. 2004. Doubling the estimate of invertebrate biomass in a rainforest canopy. Nature, 429:549551.

Fisher, B.L. 1999. Improving inventory efficiency: a case study of leaf-litter ant diversity in Madagascar. Ecological Monographs, 9: 714-731.

Fisher, B.L.; Malsch, A.K.F.; Gadagkar, R.; Delabie, J.H.C.; Vasconcelos, H.L.; Majer, J.D. 2000. Applying the ALL Protocol. In: D. Agosti, J. D. Majer, A. Tennant e T. R. Schultz (Eds). Ants: standard methods for measuring and monitoring biodiversity. Smithsonian Institution Press, Washington, DC., USA. p. 207-214.
Hölldobler, B.; Wilson, E.O. 1990. The Ants. Belknapp Press of Harvard University, Cambridge, MA. 733pp.

Kempf, W. W. 1972. Catálogo abreviado das formigas da região neotropical. Studia Entomologica, 15: 1-134.

Kim, K. C. 1993. Biodiversity, conservation and inventory: why insects matter. Biodiversity Conservation, 2: 191-214.

Hedlund, K. S. 2004. (http://www.cs.unc.edu/-hedlund/dev/ants/ catalog/.) Acesso: 10/01/2004.

Lacruz, M. S. P. 1996. Sensoriamento remoto e sistemas de Informação geográfica como subsídio para Levantamentos fisionômico-estruturais em Floresta tropical úmida - estudo de caso: Estação científica Ferreira Penna, PA. Dissertação de Mestrado, Instituto Nacional de Pesquisas Aeroespaciais/Ministério da Ciência e Tecnologia. São José dos Campos, São Paulo. 131 p.

Lattke, J. 1990. Revision del genero Gnamptogenys Mayr en Venezuela (Hymenoptera: Formicidae). Acta Terramaris, 2: 1-47.

....1995. Revision of the ant genus Gnamptogenys in the New World (Hymenoptera: Formicidae). Journal of Hymenoptera Research, 4:137-193.

Levings, S. G. e Franks, N. R. 1982. Patterns of nest dispersion in a tropical ground ant community. Ecology, 63(2):338-344.

Lindsey, P.A. e Skinner, J. D. 2001. Ant composition and activity patterns as determined by "Pitfall" trapping and other methods in three habitats in semi-arid Kayo. Journal of Arid Environment, 48:51-568.

Lobry de Bruyn, L. A. 1999. Ants as bioindicators of soil function in rural environments. Agriculture, Ecosystems e Environment, 74:425-441.

Longino, J.T. 2003. The Crematogaster (Hymenoptera, Formicidae, Myrmicinae) of Costa Rica. Zootaxa, 151:1-150.

Longino, J. T. 2005. (http://www.evergreen.edu/ants.) Acesso: $09 / 02 / 2005$

Longino, J. T., Coddington, J. e Colwell, R. K. 2002. The ant fauna of a tropical rain forest: estimating species richness tree different ways. Ecology, 83(3):689-702.

Lutinski, J.A. e Garcia, F.R.M. 2005. Análise faunística de Formicidae (Hymenoptera: Apocrita) em ecossistema degradado no município de Chapecó, Santa Catarina. Biotemas, 18(2):7386.

Mackay, W. e Mackay, E. 2003. (http://www.utep.edu/leb/ants/ Ponerinae.htm.) Acesso: 10/01/2004.

Majer, J.D.; Delabie, J.H.C. e McKenzie, N.L. 1997. Ant litter fauna of forest, forest edges and adjacent grassland in the Atlantic rain forest region of Bahia, Brazil. Insectes Sociaux, 44:255-266.

Margules, C. R. e Austin, M. P. (Eds.) 1991. Nature Conservation: Cost Effective Biological Surveys and Data Analysis. CSIRO, Australia.

Margules, C. R. e Redhead, T. D. 1995. Guidelines for using the BioRap Methodology Tools. Fyshwick, ACT, CSIRO.

Margurran, A.E. 2005. Species abundance distributions: pattern or process? Functional Ecology 19: 177-181. 
Murray, B. R. e Lepschi, B. J. 2004. Are locally rare species abundant elsewhere in their geographical range? Austral Ecology, 29:287-293.

Olson, D. M. 1991. A comparison of the efficacy of litter sifting and "Pitfall" traps for sampling leaf litter ants (Hymenoptera, Formicidae) in a tropical wet Forest, Costa Rica. Biotropica, 23(2):166-172.

Parr, C.L.; Chown, S.L. 2001. Inventory and bioindicator sampling: testing pitfall and Winkler methods with ants in a South African savanna. Journal of Insect Conservation, 5: 27-36.

Silva, R.R.; Silvestre, R. 2004. Riqueza da fauna de formigas (Hymenoptera: Formicidae) que habita as camadas superficiais do solo em Seara, Santa Catarina. Papéis Avulsos de Zoologia, 44(1): 1-11.

Soares, S.M.; Schoereder, J.H.; DeSouza, O. 2001. Processes involved in species saturation of ground-dwelling ant communities (Hymenoptera, Formicidae). Austral Ecology, 26: 187-192.

Scotland, R.; Huges, C.; Bailey, D.; Worley, A. 2003. The Big Machine and the much-maligned taxonomist. Systematics and Biodiversity, 1(2):139-143

Sörensen, L.L.; Coddington, J.A.; Sharff, N. 2002. Inventorying and estimating subcanopy spider diversity using semiquantitative sampling methods in an afromontane forest. Environmental. Entomology. 31(2): 319-330. van den Berghe, E. 1992. On pitfall trapping invertebrates. Entomological News, 103(4): 149-156.

Umetsu, F.; Naxara, L.; Pardini, R. 2006. Evaluating the efficiency of pitfall traps for sampling small mammals in the neotropics. Journal of Mammalogy, 87 (4): 757-765.

Vasconcelos, H.; Delabie, J.H.C. 2000. Ground ant communities from central Amazonia forest fragments. In: Agosti, D.; Majer, J.D.; Alonso, L. e Schultz, T. (Eds.), Sampling ground-dwelling ants: case studies from de world's rain forests. Bulletin 18. Curtin University School of Environmental Biology, Perth, Australia, p. 59-70.

Vasconcelos, H.L.; Macedo, A.C.C.; Vilhena, J.M.S. 2003. Influence of topography on the distribution of ground-dwelling ants in an Amazonian forest. Studies on Neotropical Fauna and Environment, 38(2):115-124.

Wild, A.L. 2002. The Genus Pachycondyla (Hymenoptera: Formicidae) in Paraguay. Bol. Mus. Nac. Hist. Nat. Parag., 14(1-2): 1-18.

Wilkinson 1990. SYSTAT: The system for statistics Systat, version 8.0 for Windows. Evanston, Illinois. 822pp.

Recebido em 23/08/2005

Aceito em 07/11/2007 